\section{Sepsis therapy in the next decade: seamless care from emergency to the ICU}

To the Editor: Severe sepsis: defined by inflammation (aberrant temperature and white blood cell count, tachypnea and tachycardia); suspicion of infection; and evolving organ dysfunction, is associated with an incidence, mortality and cost similar to acute coronary syndromes. ${ }^{1}$ However, sepsis has not received similar recognition or funding. Evidence suggests "golden hours" during which early and aggressive therapy can prevent progression to intractable organ failure. ${ }^{2}$ Guidelines now provide evidence-based strategies to expedite a continuum of care from the emergency department, to the ward, to the ICU. ${ }^{3}$ As such, the next decade holds great potential. Conceptually this is similar to the "chain-of-survival" recommended for cardiac disease. ${ }^{4}$ Early goal-directed therapy, early antibiotic administration, tight glycemic control, low-dose corticosteroids, consideration of recombinant protein $\mathrm{C}$, and consideration of early transfer to ICU are all likely to improve mortality. ${ }^{3}$

To promote seamless multidisciplinary care, representatives from 5 Canadian sites met to exchange ideas and learn from the world authority. ${ }^{3}$ Emergency, intensive care and internal medicine physicians along with nurses, educators and administrators discussed strategies. They agreed that the use of established protocols will help to provide optimal care delivery, germinate education and stimulate research..

We welcome correspondence, both to share our nascent protocols and to stimulate nationwide involvement. We encourage others to address their own continuum of sepsis care and learn how to facilitate early sepsis therapy while the greatest chance for recovery exists.

We thank Dr. Emanuel Rivers and the other staff members at Detroit's Henry Ford Hospital who provided the impetus to tackle this disease. It is now time for Canadian physicians to show leadership and for funding to follow.

\section{Peter G. Brindley}

Assistant Professor

Critical Care Medicine

4H1.22, University of Alberta Hospital 8440-112th St.

Edmonton AB T6G 2B7

peterbrindley@cha.ab.ca

Dennis Djogovic

Emergency Medicine

University of Alberta Hospital

Edmonton, Alta.

\section{References}

1. Angus DC, Linde-Zwirble WT, Lidicker J, Clermont G, Carcillo J, Pinsky MR. Epidemiology of severe sepsis in the United States: analysis of incidence, outcome, and associated costs of care. Crit Care Med 2001;29:1303-10.

2. Rivers E, Nyugen B, Havstad S, Ressler J, Muzzin A, Knoblich B, et al. Early-goal directed therapy in the treatment of severe sepsis and septic shock. N Engl J Med 2001;345:1368-77.

3. Dellinger RP, Carlet JM, Masur H, Gerlach H, Calandra T, Cohen J, et al. Surviving sepsis campaign guidelines for management of severe sepsis and septic shock. Crit Care Med 2004;32:858-72.

4. Guidelines 2000 for Cardiopulmonary Resuscitation and Emergency Cardiovascular Care (70-2041). Part 1: Introduction to the International Guidelines 2000 for CPR and ECC: a consensus on science. The American Heart Association in collaboration with the International Liaison Committee on Resuscitation. Circulation 2000;102(suppl 1):I1-11.

\section{Children and magnet-backed jewellery}

To the Editor: My thanks to your Jour- nal and Drs. Brown, Tomasi and Salcedo, the authors of "An attractive approach to magnets adherent across the nasal septum." This past Christmas Eve, during my first year as an attending, I was providing solo coverage in the pediatric emergency department at one of our community hospitals. An 11-year-old girl presented with - yes — two small jewellery magnets magnetized across her nasal septum. She was mildly uncomfortable and extremely anxious.

For me, this was classic pediatric emergency lore - I'd heard about it, had never seen it, and was not quite sure how to manage it. I asked for a magnet, and was given the large 4-in $\times 6$-in block from the adult code cart; I felt this was likely to be difficult to get into the patient's nose. A literature search revealed the article in your Journal. As suggested, I inserted the metal handle of a bayonet forceps into the nostril to effectively utilize the magnetic properties of the jewellery; as a variation on the method described, I simultaneously placed the metal handle of a separate pair of forceps into the other nostril. Each magnet adhered readily to the instrument in each nostril, and they were readily withdrawn, with instant relief of both pain and anxiety — the patient's and mine.

\section{Marisa Brett-Fleegler, MD}

Attending

Division of Emergency Medicine

Children's Hospital Boston

Boston, Mass.

\section{Reference}

1. Brown L, Tomaso A, Salcedo G. An attractive approach to magnets adherent across the nasal septum. Can J Emerg Med 2003;5(5):356-8. 